\title{
Real-Time Track Reallocation for Emergency Incidents at Large Railway Stations
}

\author{
Wei Liu, Xiaoning Zhu, and Liujiang Kang \\ School of Traffic and Transportation, Beijing Jiaotong University, Beijing 100044, China \\ Correspondence should be addressed to Liujiang Kang; 243525771@qq.com
}

Received 16 September 2015; Revised 13 November 2015; Accepted 16 November 2015

Academic Editor: Egidijus R. Vaidogas

Copyright ( 2015 Wei Liu et al. This is an open access article distributed under the Creative Commons Attribution License, which permits unrestricted use, distribution, and reproduction in any medium, provided the original work is properly cited.

\begin{abstract}
After track capacity breakdowns at a railway station, train dispatchers need to generate appropriate track reallocation plans to recover the impacted train schedule and minimize the expected total train delay time under stochastic scenarios. This paper focuses on the real-time track reallocation problem when tracks break down at large railway stations. To represent these cases, virtual trains are introduced and activated to occupy the accident tracks. A mathematical programming model is developed, which aims at minimizing the total occupation time of station bottleneck sections to avoid train delays. In addition, a hybrid algorithm between the genetic algorithm and the simulated annealing algorithm is designed. The case study from the Baoji railway station in China verifies the efficiency of the proposed model and the algorithm. Numerical results indicate that, during a daily and shift transport plan from 8:00 to 8:30, if five tracks break down simultaneously, this will disturb train schedules (result in train arrival and departure delays).
\end{abstract}

\section{Introduction}

To solve the track allocation problem in a railway station, a conflict-free route must be found for each arrival/departure train. This optimization problem, known as the train routing problem or the train platform problem, is one of the basic scheduling problems for railway companies. In terms of complexity, this problem is NP-hard [1]. Nevertheless, it can be modeled and solved using operation research techniques. Due to the signal problem, frequent use, or other reasons, available tracks at a railway station may break down (may be unavailable). In addition, track incidents are generally uncertain and often disturb train schedules such that trains will be delayed if station tracks are not effectively reallocated. In this paper, we consider the problem of real-time reallocation of trains to available tracks (except accidental tracks) at a large railway station, given one daily and shift transport timetable and the structural and operational constraints.

There are many existing studies that solve the track allocation problem. Zwaneveld et al. [2] proposed a node packing formulation, and infrastructure capacity requirements were developed to evaluate future demand for rail transportation.
To improve the solution method, Zwaneveld et al. [3] conducted an extension of [2]. Zwaneveld et al. assumed that trains prefer certain paths to others and this problem was formulated as a weighted node packing problem. A branchand-cut solution approach was adopted to solve this NP-hard problem.

Cardillo and Mione [4], Billionnet [5], and Carey and Carville [6] studied a version of the problem termed train platform problem (TPP). Cardillo and Mione [4] proposed a heuristic algorithm with a backtracking method. The work of [5] demonstrated how the TPP models could be strengthened through the addition of clique inequalities. Carey and Carville [6] studied the problem at busy complex stations and developed scheduling heuristics analogous to train operators using the manual method.

TPP is affected by the structure of stations and yards, the bottleneck section structure, station operations, train timetables, train punctuality, and many other factors among which the train working diagram (TWD) plays an important role because it determines the start and end times of tracks utilized at railway stations [7]. Barber et al. [8] presented a set of heuristics for a constraint-based TWD tool and formulated 
TWD as a constraint optimization problem. According to TWD and train routing in stations, Kang et al. [7] combined TPP with the station bottleneck carrying capacity. A case study that focused on bottleneck section optimization in passenger stations illustrated that TPP affects not only bottleneck capacity but also station capacity. D'Ariano et al. [9] introduced a new concept aimed at less planning on the timetable and solving more intertrain conflicts to improve punctuality without decreasing line capacity. A detailed model for conflict resolution was illustrated, and different algorithms based on the alternative graph formulation were analyzed in their work. Heydar et al. [10] investigated the capacity of a single track, bidirectional rail line that adheres to a cyclic timetable.

When probabilities for unexpected events are estimated, efficiency is a major concern and can be addressed through multilevel splitting or staged simulation [11]. After major service disruption in the railway operational system, dispatchers need to generate a series of train meet-pass plans at different decision times of the rescheduling stage to recover the impacted train schedule from current and future disturbances and minimize the expected additional delay under different forecasted operational conditions. Thus, railway controllers play a pivotal role in the service recovery of normal rail system operations when accidents occur. Cheng and Tsai [12] adopted Taiwan's railway system to diagnose railwaycontroller-perceived competence when facing diverse tasks during incidents and accidents that were derived from a proposed conceptual model. Belmonte et al. [13] presented an application of functional resonance accident models (FRAM) for the safety analysis of complex sociotechnological systems, that is, systems with not only technological components but also human and organizational components. The examples illustrated the principal advantages of FRAM in comparison to classical safety analysis models, which allowed true multidisciplinary cooperation between specialists from the different domains involved.

Operationally robust solutions are usually synonymous with compact, nonoverlapping routes with little or no intraroute crossover [14]. Railway companies are also interested in schedule reliability (assessed by means of cancelling the number of operating trains) and robustness (the ability to resist schedule perturbations). Intuitively, a more robust track allocation schedule is less likely to propagate delays to the following trains. Clearly, an efficient use of the railway infrastructure and the prospects of recovery cause TPP to become a more complex optimization problem in engineering theory and practice.

The aforementioned studies mainly focus on the track allocation optimization problem. After the track capacity breakdown(s) at a railway station, train dispatchers need to generate appropriate track reallocation plans to recover the impacted train schedule and minimize the expected total train delay time under stochastic scenarios. As a result, reallocating tracks in railway stations for emergency incidents to ensure service remains a significant issue. A model that can be exactly solvable by a global optimization method is also desired.
On the basis of station track incidents, a model considering the minimum average use time of bottleneck sections is proposed to reduce technical process time and improve the antijamming capacity. The bottleneck and station carrying capacity can be improved simultaneously by tapping the potential and more effective occupation of normal tracks and bottleneck equipment. The contributions of this paper are listed as follows:

(1) A mathematical programming model is proposed to describe TPP after track incidents, which fully considers the tight capacity of station bottlenecks when some tracks break down.

(2) Virtual trains are introduced to represent track incidents. The novelty of this formulation is the use of virtual trains to occupy these unavailable tracks. In the Baoji passenger station, an incident in which five tracks break down simultaneously will lead to the delay of trains' arrival/departure.

(3) Genetic simulated annealing (GSA) focused on TPP is adopted. In addition, a genuine case in China is studied, and the station track allocation plan is given. The carrying capacity of the left side bottleneck is approximately 1.29 times more than the right side bottleneck.

This paper will be organized as follows. First, the model formulation section draws the TPP model out. In addition, the GSA of TPP is developed in the solution algorithm section. Furthermore, a real case from China is illustrated in Case Study. Finally, the Conclusions summarize this paper and discuss the future study.

\section{Track Reallocation Model}

2.1. Notations. Notations in this paper are as follows

Sets

I: set of groups of turnouts (GTs), $i \in I, I=$ $\{i / i=1,2, \ldots, n\}$, where $n$ is the total number of GTs. Herein, turnout is also called switch in stations.

$I_{L}$ : set of GTs in the left station bottleneck, which consists of the number of GTs.

$I_{R}$ : set of GTs in the right station bottleneck.

$J$ : set of trains, $j \in J, J=\{j / j=1,2, \ldots, m\}$, where $m$ is the total number of trains scheduled within TWD.

$R$ : set of reception-departure tracks, $r \in R, R=\{r / r=$ $1,2, \ldots, l\}$, where $l$ is the total number of receptiondeparture tracks at a passenger station.

\section{Parameters}

$T_{S}$ : security interval time between two neighboring trains that occupy the same track.

$t_{r j}^{S}$ : starting time of train $j$ occupying track $r$.

$t_{r j}^{E}$ : ending time of train $j$ occupying track $r$.

$t_{i j}$ : traversing time of train $j$ occupying GT $i$. 


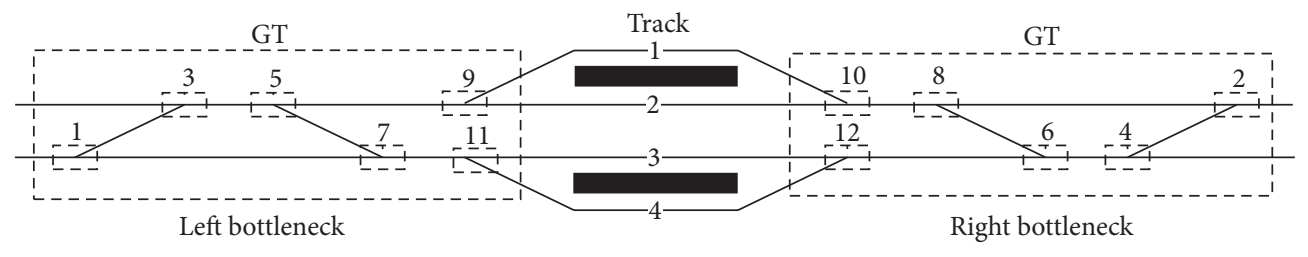

FIGURE 1: A sample station structure.

\section{Variables}

$x_{i j}= \begin{cases}1, & \text { if } \\ 0, & \text { train } j \text { occupies GT } i\end{cases}$

$x_{r j}= \begin{cases}1, & \text { if } \\ 0, & \text { train } j \text { stays at track } r\end{cases}$

$T_{j}$ : the total time cost of GTs by train $j$.

$T_{L}$ : the total time cost of GTs in the left bottleneck (detailed illustration of the left bottleneck can be found in Figure 1), $T_{L}=\sum_{i} \sum_{j} t_{i j}$.

$T_{R}$ : the total time cost of GTs in the right bottleneck (detailed illustration of the right bottleneck can be found in Figure 1), $T_{R}=\sum_{i} \sum_{j} t_{i j}$.

2.2. Basic Assumptions. To solve and optimize TPP, several assumptions are used throughout this paper to simplify practical cases:

(1) Train arrival/departure times at/from the station are collected according to the existing timetable and cannot be changed. That is, there is no train shifting from the original timetable. In this case, the track reallocation plan is ordered by train arrival-departure times.

(2) Different trains traverse the same GT with equal time. This assumption eliminates the complexity of considering train types (e.g., train formation, train ranks). Thus, the total occupation time of a certain route composed of GTs can be accumulated.

(3) The security interval time $\left(T_{S}\right)$ is assumed as a constant. Generally, there are different ranks of trains passing through stations, for example, passenger trains and freight trains. Obviously, $T_{S}$ varies between different ranks of trains for their unique braking performance. This assumption can help substantially reduce the complexity of the model.

2.3. Model Formulations. To better explain the model formulations, a sample station is illustrated in Figure 1. There are four tracks and twelve GTs numbered from 1 to 4 and from 1 to 12 , respectively, in the sample station. In Figure 1 , the left bottleneck section contains six GTs, which are marked with odd numbers. On the contrary, the right bottleneck section consists of six even numbered GTs.

2.3.1. Constraints of TPP and Track Accidents. Operational constraints in a passenger station set limits on the track allocations of trains. To fulfill the service requirements and enable safety, the following constraints should be satisfied.

One Time, One GT, and One Train. For each GT $i \in I$ and $j \in J$, constraints (1) set the uniqueness occupation of each GT in a certain period of time punctually, where $x_{i j}$ is a binary variable representing whether train $j$ occupies GT $i$. A fixed GT cannot be traversed by other trains until it is released by the preceding train. Thus, during the periods of $\left[t_{r j}^{S}-T_{s}, t_{r j}^{S}\right]$ and $\left[t_{r j}^{E}, t_{r j}^{E}+T_{s}\right]$, where $t_{r j}^{S}$ and $t_{r j}^{E}$ represent the starting and ending times of train $j$ occupying track $r$, constraints (1) should be satisfied:

$$
\begin{aligned}
& {\left[t_{r j}^{S}-T_{s}, t_{r j}^{S}\right]: \sum_{j=1}^{m} x_{i j} \leq 1,} \\
& {\left[t_{r j}^{E}, t_{r j}^{E}+T_{s}\right]: \sum_{j=1}^{m} x_{i j} \leq 1 .}
\end{aligned}
$$

Obviously, if a train pulls in from the left bottleneck and stays on track 1 in Figure 1, it will traverse GTs [2, 3, 5, 8-10]. At the same time, if another train also pulls in from the left bottleneck, it cannot stay on track 2 because GTs [2, 3, 5, 8-10] have been occupied. However, this train can sit on track 3 or track 4 because GTs $[1,4,6,7,11,12]$ are free at that moment.

One Time, One Track, and One Train. $x_{r}$ is a binary variable. $x_{r}=1$ means track $r$ breaks down, and $x_{r}=0$ means track $r$ can receive trains. Constraint (2) indicates that, during the period of $\left[t_{r j}^{S}, t_{r j}^{E}\right]$, track $r$ is reserved for train $j$. Apart from this period, track $r$ is released. Thus, constraint (2) examines the track state and limits the unique utilization of station tracks as well:

$$
\left[t_{r j}^{S}, t_{r j}^{E}\right]: \sum_{j=1}^{m} x_{r j} \leq\left(1-x_{r}\right) .
$$

When $x_{r}$ is equal to one, we have $\sum_{j=1}^{m} x_{r j} \leq\left(1-x_{r}\right)=0$. This track breaks down and is occupied by one virtual train. On the contrary, when $x_{r}$ is equal to zero, the right hand side $\sum_{j=1}^{m} x_{r j} \leq\left(1-x_{r}\right)=1$ and track $r$ is ready for allocation. With the binary variable $x_{r}$ so defined, track incidents are described accurately. 


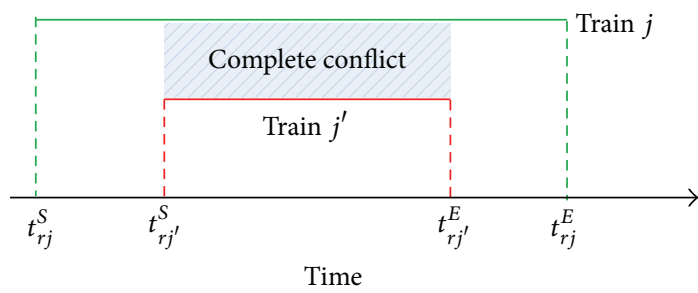

FIgURE 2: Complete conflict. Train $j$ occupies track $r$ from time $t_{r j}^{S}$ to time $t_{r j}^{E}$, and train $j^{\prime}$ occupies track $r$ from time $t_{r j^{\prime}}^{S}$ to time $t_{r j^{\prime}}^{E}$, where $t_{r j}^{S} \leq t_{r j^{\prime}}^{S} \leq t_{r j^{\prime}}^{E} \leq t_{r j}^{E}$.

One Time, One Train, and One Track. Clearly, each coming train should be allocated with only one track. In the period of $\left[t_{r j}^{S}, t_{r j}^{E}\right]$, train $j$ will stay on track $r$. Hence,

$$
\left[t_{r j}^{S}, t_{r j}^{E}\right]: \sum_{r=1}^{l} x_{r j}=1 .
$$

One Track, Two Trains, and Two Conflicts. A two-train conflict or greater can be decomposed by two trains. There are two types of conflicts on a track that must be avoided: complete conflict and partial conflict as referred to in Figures 2 and 3, respectively. To fulfill the requirements, there is a minimum security interval time, denoted by $T_{S}$, for two trains pulling on the same track. Figure 4 shows how two trains can stay on one identical track successively.

According to Figure 4, a conflict avoidance constraint can be built as (4); that is, one train cannot pull on to track $r$ until the preceding train has departed from track $r$ for at least $T_{S}$ min. Hence, $\forall j \in J, j^{\prime} \in J$, and $r \in R$,

$$
x_{r j^{\prime}} \cdot T_{r j^{\prime}}^{S}-x_{r j} \cdot T_{r j}^{E} \geq T_{S} .
$$

2.3.2. Objective Function of TPP. Many other urgent problems may be induced under sudden incidents on station reception-departure tracks, such as a delay in the train's arrival and departure and decreasing the carrying capacity of station bottleneck sections. These problems are brought about directly from one phenomenon: decreasing the number of available station tracks. In this case, station operators must repair tracks as quickly as possible and reallocate tracks effectively with a limited number of available tracks.

Figure 5 illustrates the flow of track reallocation after station track incidents. First, if the tracks have incidents, then the station capacity will definitely decrease. In this situation, the station bottleneck capacity also decreases because some tracks and GTs are frozen. Then, the predetermined routes (consisting of GTs) are changed and a new track reallocation plan is formulated.

The occupation time of each route to each track can be accumulated by GTs. For any train $j$, the traversing time of GTs is calculated in (5). In addition, different routes consist of different GTs. The average occupation time of each GT is used to express the cost of the route. Clearly, the less average occupation time a GT has, the better the route we choose.

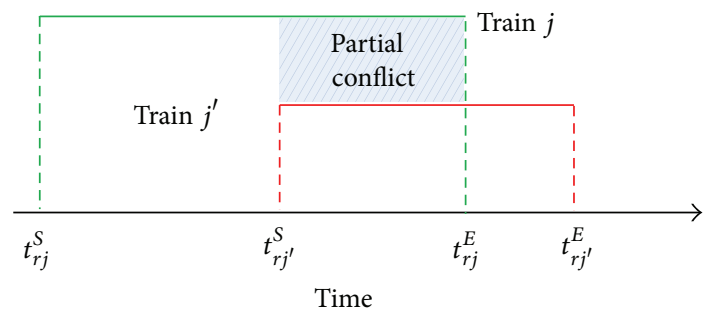

FIgure 3: Partial conflict. Train $j$ occupies track $r$ from $t_{r j}^{S}$ to $t_{r j}^{E}$, and train $j^{\prime}$ occupies track $r$ from $t_{r j^{\prime}}^{S}$ to $t_{r j^{\prime}}^{E}$, where $t_{r j}^{S} \leq t_{r j^{\prime}}^{S} \leq t_{r j}^{E} \leq t_{r j^{\prime}}^{E}$.

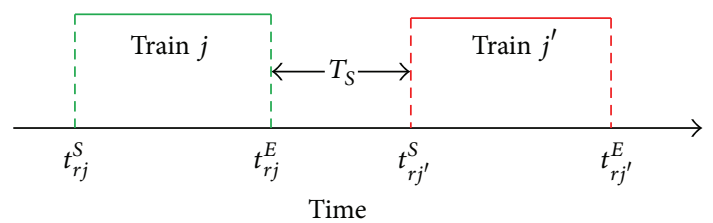

FIgURE 4: Conflict avoidance. Train $j$ occupies track $r$ from $t_{r j}^{S}$ to $t_{r j}^{E}$, and train $j^{\prime}$ occupies track $r$ from $t_{r j^{\prime}}^{S}$ to $t_{r j^{\prime}}^{E}$, where $t_{r j}^{S} \leq t_{r j}^{E} \leq$ $t_{r j^{\prime}}^{S}-T_{s} \leq t_{r j^{\prime}}^{E}-T_{s}$.

Therefore, (6) evaluates the average occupation time of GTs in a route. Therefore,

$$
\begin{aligned}
T_{j} & =\sum_{i=1}^{n} \sum_{j=1}^{m} x_{i j} \cdot t_{i j}, \\
f & =\frac{\sum_{i=1}^{n} \sum_{j=1}^{m} x_{i j} \cdot t_{i j}}{\sum_{i=1}^{n} \sum_{j=1}^{m} x_{i j}} .
\end{aligned}
$$

As we know, if some tracks are in incidents, the carrying capacity of the tracks will decrease immediately. In addition, the capacity of the bottleneck sections is also reduced. Therefore, the objective function of our model should pursue the minimum average use time of GTs in bottleneck sections that constitute different routes and lead to normal and abnormal station tracks. For all $i \in I, j \in J, j^{\prime} \in J$, and $r \in R$,

$$
\begin{array}{ll}
f=\min & \frac{\sum_{i=1}^{n} \sum_{j=1}^{m} x_{i j} \cdot t_{i j}}{\sum_{i=1}^{n} \sum_{j=1}^{m} x_{i j}} \\
\text { s.t. } \quad\left[t_{r j}^{S}-T_{s}, t_{r j}^{S}\right]: \sum_{j=1}^{m} x_{i j} \leq 1 \\
& {\left[t_{r j}^{E}, t_{r j}^{E}+T_{s}\right]: \sum_{j=1}^{m} x_{i j} \leq 1} \\
& {\left[t_{r j}^{S}, t_{r j}^{E}\right]: \sum_{j=1}^{m} x_{r j} \leq\left(1-x_{r}\right)} \\
& {\left[t_{r j}^{S}, t_{r j}^{E}\right]: \sum_{r=1}^{l} x_{r j}=1} \\
& x_{r j^{\prime}} \cdot T_{r j^{\prime}}^{S}-x_{r j} \cdot T_{r j}^{E} \geq T_{S} .
\end{array}
$$




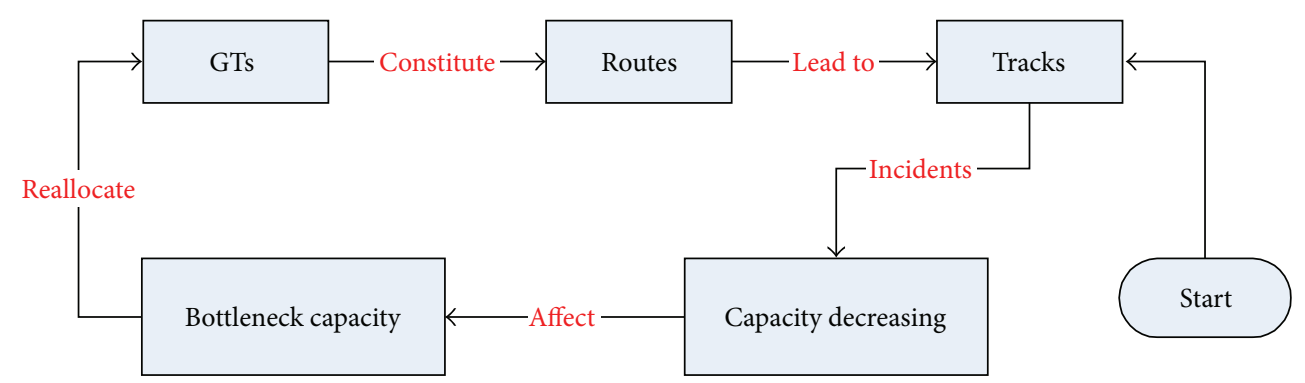

FIGURE 5: Track reallocation flow.

\section{Solution Algorithm and Sample Test}

Artificial intelligence algorithms have been widely adopted to solve NP-hard problems. Gandibleux et al. [15] proposed Ant Colony Optimization heuristics to solve the track allocation problem. As evolution of [15], the algorithm described in [16] improved the local search heuristic and stopping criterion. The node packing formulation used in [17] found robust routing for the train routing problem. In addition, [17] adopted the fixed-point iteration heuristic to exploit the clique structure. The improved research by [18] used the same fixed-point heuristic to consider both deterministic and probabilistic robust problem. Similarly, [7, 19] studied the track allocation problem at railway stations using the simulated annealing (SA) algorithm.

Studies $[20,21]$ designed a stochastic search scheme based on SA and the genetic algorithm (GA). For a nonconvex optimization system, GA may suffer from premature convergence on a local optimum. Escaping from local optima is accomplished with an SA search scheme, which is a stochastic process. Intervention in the SA is accomplished with a GA search scheme that provides promising search locations or directions when an SA search slows down or "freezes." Considering the nice compatibility of both GA and SA algorithms, a genetic simulated annealing (GSA) algorithm approach is created. Herein, the GSA algorithm will be briefly reviewed.

3.1. Genetic Simulated Annealing. SA starts from an initial solution at a high temperature and makes a series of changes according to an annealing schedule. For each change, an objective value $f_{\text {new }}$ ( $f_{\text {new }}$ becomes $f_{\text {old }}$ after an iteration) is obtained. The difference between the objective values $(\Delta f=$ $\left.f_{\text {new }}-f_{\text {old }}\right)$ is calculated after each iteration. If $\Delta f \leq 0$, the new solution is accepted with probability $\rho=1$. On the contrary, it is accepted with a small probability $\rho$, which can be tracked as $\rho=\exp (-\Delta f / T)$, where $T$ is the current temperature parameter. Thus, $\mathrm{SA}$ avoids being trapped in a local optimum [22]. Moreover, at each temperature, the above process will repeat $L$ times, where $L$ represents the Markov length. The temperature $T$ is gradually decreased by cooling coefficient $\omega$, where $T$ is defined as $T=T \times \omega(0<\omega<1)$. SA terminates either when the optimum solution is obtained or when the initial temperature decreases to the given value [7].
A matrix $\left[x_{11}, x_{21}, \ldots, x_{r 1}, \ldots, x_{l 1} ; x_{12}, x_{22}, \ldots, x_{r 2}, \ldots, x_{l 2}\right.$
$\left.\ldots ; x_{1 j}, x_{2 j}, \ldots, x_{r j}, \ldots, x_{l j} ; \ldots ; x_{1 m}, x_{2 m}, \ldots, x_{r m}, \ldots, x_{l m}\right]$ forms the genes of a chromosome in the algorithm. Here, from $j=1: m, r=1: l$, each gene $x_{r j}=1$ represents the notion that train $j$ stays on track $r$. The matrix above is a unit matrix and ensures that each coming train has a track. A common operator used in GA is crossover, which generates chromosomes [23]. A replacing method is adopted in the crossover operation. Taking the sample station as an example (four tracks), the track for a certain train is replaced by the track for the same train in another chromosome (see Figure 6), where the $x$-axis represents trains and the $y$-axis represents the timeline. The mutation operation changes the track for one train randomly. If the generated chromosome is unavailable, then it will be deleted.

The following GSA searches solutions for the track reallocation problem in railway stations. The detailed algorithmic steps are described as follows.

Step 1 (initialization). (1.1) Set initial parameters: population size $p(p=1000)$, iteration mark $k:=0$, initial temperature $t_{k}:=100$, lowest temperature $\tau=0.01$, and cooling coefficient $\omega=0.95$.

(1.2) Read the timetables for the arrival-departure trains.

Step 2 (creating and selecting new chromosome). (2.1) Update $k=k+1$.

(2.2) If $t_{k}:=\tau$, stop; otherwise, turn to 2.2 .

(2.3) Obtain a new chromosome $j$ from each old chromosome $i \in \operatorname{POP}(k)$ from the crossover operation to chromosome $i$.

(2.4) Calculate $\sigma(j)-\sigma(i)$, where $\sigma(j)$ and $\sigma(i)$ are objective values of chromosomes $j$ and $i$, respectively.

(2.5) If $\sigma(j)-\sigma(i) \leq 0$, chromosome $j$ replaces chromosome $i$; otherwise, chromosome $j$ replaces chromosome $i$ by probability $\rho=\exp \left((f(i)-f(j)) / t_{k}\right)$.

(2.6) Repeat steps 2.2 through 2.5 until a new population $\operatorname{POP} 1(k)$ is generated.

(2.7) Use the mutation operation to population POP1 $(k)$. Check the generated solutions with constraints in (7) and accept them if they are available. Otherwise, delete them.

(2.8) Find the best solution in POP1 $(k)$ by comparing objective values. 


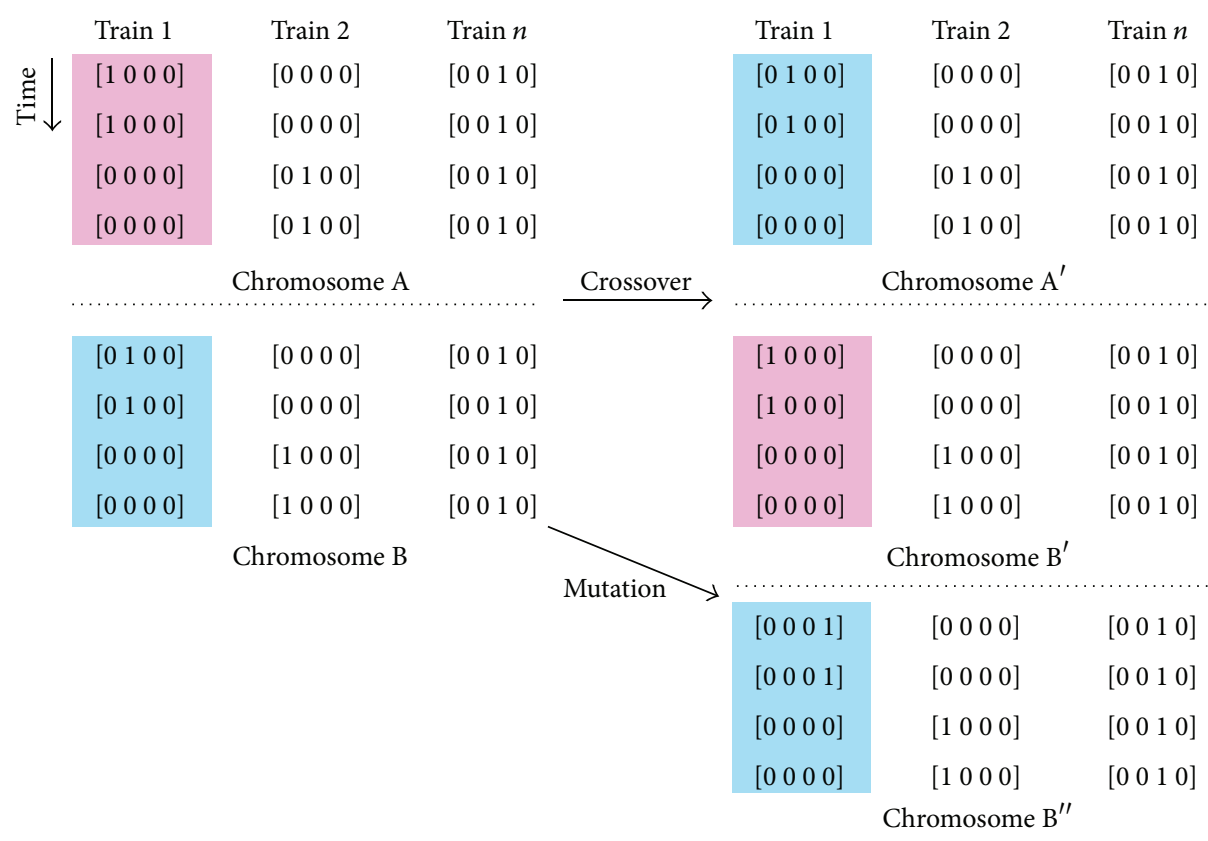

Crossover: two different chromosomes generate two new chromosomes

Mutation: one chromosome generates a new chromosome

FIgURE 6: Samples of crossover and mutation operations.

Step 3 (stop or do not stop). 3.1 If temperature $t_{k} \leq \tau$, stop; otherwise, $t_{k}=t_{k} \cdot \omega$. Return to Step 2 .

3.2. Sample Test. A sample station in Figure 1 is used to verify the feasibility of the proposed model. Table 1 shows the different components for the routes and the corresponding occupation time of the GTs. For example, GTs (3, 5, 9/10, 8,2 ) consist of the path to reception-departure track 1 . The corresponding occupation times of the above GTs are $(2,2$, $2 / 2,2,2$ ) seconds.

Table 2 gives the arrival and departure times of trains that should be dispatched in this station. There are six trains from 8:00 to 8:25 among which three trains pull in from the right bottleneck section and three pull in from the left bottleneck section. Figure 7 depicts the timetable intuitively. As observed, T2, T4, T5, and T6 arrive/depart at/from the station simultaneously. In other words, these four trains will occupy the station bottlenecks at 8:15, which means this station stays in the thick of the bottleneck capacity at this time.

Experiments are tested on a personal computer with an Intel Pentium $42.80 \mathrm{GHz} \mathrm{CPU}$ and $2 \mathrm{~GB}$ RAM. The track allocation optimizing results are given in Table 3 , when $\sum_{i \in I} t_{i j} / \sum_{i \in I} x_{i r}$ of tracks 1 and 2 are smaller than those of tracks 3 and 4 . Thus, tracks 1 and 2 have been occupied twice, while tracks 3 and 4 are only used once.

We also simulate the track accidental experiment (track 1 is randomly selected by the programming). Computing results are shown in Table 4 . When we simulate two track incidents simultaneously, a feasible reallocation plan could not be returned because the bottleneck capacity is not large enough to receive all trains. The computer simulation
TABLE 1: Component GTs of each track in Figure 1.

\begin{tabular}{lcccc}
\hline Track & $\begin{array}{c}\text { Groups of } \\
\text { turnouts }\end{array}$ & $\sum_{i \in I} x_{i r}$ & $t_{i j}(\mathrm{sec})$ & $\sum_{i \in I} t_{i j} / \sum_{i \in I} x_{i r}(\mathrm{sec})$ \\
\hline 1 & $3,5,9 / 10,8,2$ & $3 / 3$ & $2,2,2 / 2,2,2$ & 2 \\
2 & $3,5,9 / 10,8,2$ & $3 / 3$ & $2,2,2 / 2,2,2$ & 2 \\
3 & $1,7,11 / 12,6,4$ & $3 / 3$ & $3,3,3 / 3,3,3$ & 3 \\
4 & $1,7,11 / 12,6,4$ & $3 / 3$ & $3,3,3 / 3,3,3$ & 3 \\
\hline
\end{tabular}

indicates that if there are two or more tracks that are not ready for service, trains cannot stay on the tracks according to the existing timetable. The track capacity decreases and some trains are delayed for arrival.

\section{Case Study}

This section illustrates the quality of the proposed model for a real case in China. Figure 8 shows the structure of the Baoji railway station in China. There are two bottleneck sections in this station: the left bottleneck section and the right bottleneck section. The left bottleneck is made up of 13 GTs that are marked with odd Arabic numbers, and the right bottleneck is made of 14 even GTs. Eleven receptiondeparture tracks, numbered from 1 to 11, are in the middle of the station. Here, the data sources for the computation come from the Xian Railway Administration, and the detailed TWD is given in Table 5 .

As seen from Table 5, there are 30 trains that are scheduled in this daily and shift transport plan (from approximately 8:00 to 10:00). For example, train T22 arrives at 8:09 and departs at 8:22. The Direction column indicates that train T22 
TABLE 2: TWD of trains.

\begin{tabular}{lccccccc}
\hline Train & Direction & Reception & Departure & Train & Direction & Reception & Departure \\
\hline T1 & Right & $8: 00: 00$ & $8: 10: 00$ & T2 & Left & $8: 00: 00$ & $8: 15: 00$ \\
T3 & Right & $8: 00: 00$ & $8: 05: 00$ & T4 & Left & $8: 10: 00$ & $8: 15: 00$ \\
T5 & Right & $8: 10: 00$ & $8: 15: 00$ & T6 & Left & $8: 15: 00$ & $8: 25: 00$ \\
\hline
\end{tabular}

TABLE 3: Track allocation results of sample station.

\begin{tabular}{lccccccc}
\hline Train & Track & Reception & Departure & Train & Track & Reception & Departure \\
\hline T1 & 3 & $8: 00: 00$ & $8: 10: 00$ & T2 & 1 & $8: 00: 00$ & $8: 15: 00$ \\
T3 & 2 & $8: 00: 00$ & $8: 05: 00$ & T4 & 2 & $8: 10: 00$ & $8: 15: 00$ \\
T5 & 4 & $8: 10: 00$ & $8: 15: 00$ & T6 & 1 & $8: 15: 00$ & $8: 25: 00$ \\
\hline
\end{tabular}

TABLE 4: Track reallocation results.

\begin{tabular}{lccccccc}
\hline Train & Track & Arrival & Departure & Train & Track & Arrival & Departure \\
\hline T1 & 4 & $8: 00: 00$ & $8: 10: 00$ & T2 & 3 & $8: 00: 00$ & $8: 10: 00$ \\
T3 & 2 & $8: 00: 00$ & $8: 05: 00$ & T4 & 2 & $8: 15: 00$ \\
T5 & 4 & $8: 10: 00$ & $8: 15: 00$ & T6 & 2 & $8: 15: 00$ & $8: 25: 00$ \\
\hline
\end{tabular}

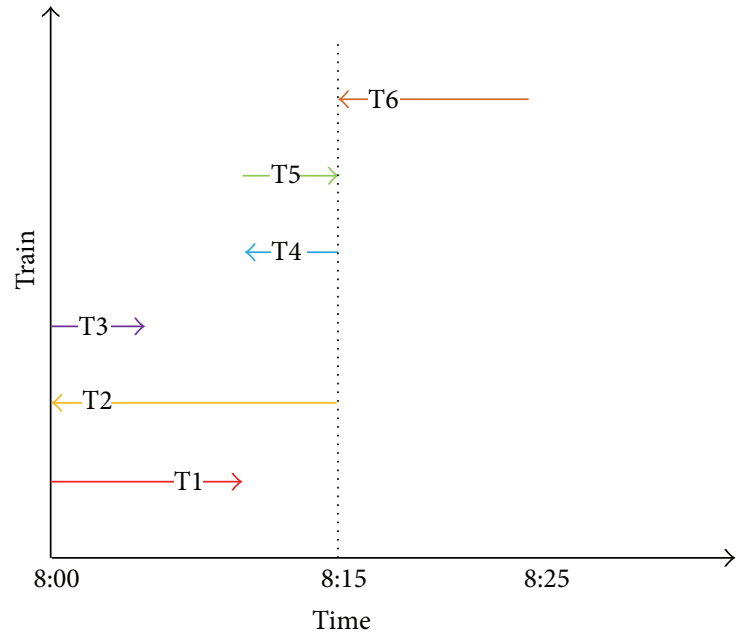

FIGURE 7: Train arrival-departure time axes.

pulls in from the right bottleneck section and pulls out from the opposite bottleneck.

In Table 6, each row gives the component GTs of a station route from track 1 to track 11 . The occupation time of each route is calculated according to Table 7 , which shows the occupation time of each GT. Here, safety interval times have been taken into consideration [24].

4.1. Unavailable Tracks Simulation. Some virtual trains are introduced to solve the simulation work. It is undoubted that if a certain track is unavailable because of incidents, this track cannot be occupied until it recovers. According to different accident degrees, different repairing times are required. Therefore, the track repairing time is represented by the track occupation time of a virtual train. Actually, each track has a hidden virtual train, which will be activated as long as the track remains unavailable. Figure 9 illustrates how virtual trains simulate the unavailable tracks. TWD is transformed into a track occupation timeline. The dotted lines represent virtual trains, and the solid lines are real trains with train running directions. Train numbers are marked above the lines with the tracks in brackets. One virtual train will occupy a track when this track breaks down. The virtual train will evacuate when the track recovers.

4.2. Optimization Results. Four accidental tracks are randomly selected by the computer, that is, tracks $2,3,8$, and 10. Track 3 and track 8 are unavailable from 8:00 to 9:00, while track 1 and track 10 are unavailable from 9:00 to 10:00. In this case, the optimization results are shown in Table 8. Clearly, no train should stay on tracks 3 and 8 between 8:00 and 9:00. Additionally, tracks 1 and 10 are not occupied between 9:00 and 10:00. Figure 10 shows the track occupation frequency and route occupation time (cost) in a line chart, where "cost" means the necessary occupation time (min) of GTs with respect to a certain track. Compared with the original allocation results, the total cost of the optimal results is reduced from 64.3 to 62.2 in $30 \mathrm{~min}$ (Table 9).

It is easy to find that the higher the route costs, the smaller the occupation frequency of the corresponding track is. For example, tracks 10, 5, 9, and 7 have been occupied four times, while tracks 8 and 11 are occupied only once because track 8 is unavailable from 8:00 to 9:00 and because its time cost reaches up to 2.3. For instance, track 10 has been occupied four times in one hour. This is mainly because its time cost is only 1.833 , which is the least among all station tracks.

4.3. Limited Carrying Capacity. According to the train arrival times in Table 8, different track allocation plans are given by discrete algorithm parameters. Table 10 lists the specific values of the left and right bottlenecks after analyzing two bottleneck capacities under each given plan. As seen, the program has been run by separate iterations (from 60 to 
TABLE 5: TWD of trains.

\begin{tabular}{|c|c|c|c|c|c|c|c|}
\hline Train & Direction & Arrival & Departure & Train & Direction & Arrival & Departure \\
\hline T22 & Right & 8:09:00 & $8: 22: 00$ & K375 & Left & $8: 24: 00$ & $8: 31: 00$ \\
\hline T75 & Left & $9: 12: 00$ & 9:22:00 & D5082 & Right & $8: 41: 00$ & 9:11:00 \\
\hline T117 & Left & $9: 42: 00$ & 9:52:00 & $\mathrm{T} 23$ & Left & 8:09:00 & 8:22:00 \\
\hline T193 & Left & $8: 29: 00$ & 8:33:00 & T116 & Right & 9:42:00 & 9:52:00 \\
\hline T223 & Left & 8:12:00 & 8:22:00 & T192 & Right & 8:29:00 & 8:33:00 \\
\hline K621 & Left & 9:04:00 & 9:12:00 & T222 & Right & $8: 12: 00$ & $8: 22: 00$ \\
\hline 10176 & Right & $9: 24: 00$ & $9: 29: 00$ & K624 & Right & 9:04:00 & 9:12:00 \\
\hline 10452 & Right & 9:14:00 & 9:22:00 & 10175 & Left & 9:24:00 & 9:29:00 \\
\hline 10454 & Right & $9: 16: 00$ & $9: 46: 00$ & 10420 & Right & 8:08:00 & $8: 19: 00$ \\
\hline 10448 & Right & 8:08:00 & 8:38:00 & 10450 & Right & 8:30:00 & 9:00:00 \\
\hline 1486 & Right & $9: 27: 00$ & $9: 40: 00$ & 10456 & Right & $9: 32: 00$ & 10:02:00 \\
\hline T7 & Left & $9: 35: 00$ & $9: 45: 00$ & D5081 & Left & $8: 51: 00$ & 9:21:00 \\
\hline 1150 & Right & $9: 35: 00$ & 10:00:00 & 1147 & Left & $9: 35: 00$ & 10:00:00 \\
\hline 2669 & Left & $9: 20: 00$ & 9:50:00 & K245 & Left & 8:39:00 & 8:49:00 \\
\hline $\mathrm{K} 248$ & Right & 8:39:00 & 8:49:00 & K378 & Right & 8:24:00 & 8:31:00 \\
\hline
\end{tabular}

TABle 6: Component GTs of each train track.

\begin{tabular}{lcccc}
\hline Track & Groups of turnouts & $\sum_{i \in I} x_{i r}$ & $\sum_{i \in I} t_{i j}$ & $8 / 8$ \\
\hline 1 & $11,13,21 / 16,18,26,28$ & $3 / 4$ & $\sum_{i \in I} t_{i j} / \sum_{i \in I} x_{i r}$ \\
2 & $11,13,21 / 16,18,26,28$ & $3 / 4$ & $8 / 8$ & 2.286 \\
3 & $11,13,21 / 6,8,10,20,24,28$ & $3 / 6$ & $8 / 13$ & $7 / 8$ \\
4 & $11,13,23 / 16,18,20,24$ & $3 / 4$ & $9 / 10$ & 2.286 \\
5 & $11,13,15,23,25 / 6,8,10,12,22$ & $5 / 5$ & $9 / 10$ & 2.333 \\
6 & $1,7,9,17 / 6,8,10,12,14$ & $4 / 5$ & $6 / 10$ & 2.111 \\
7 & $1,3,9 / 6,8,10,12,14$ & $3 / 5$ & $10 / 13$ & 2.000 \\
8 & $3,5,7,9 / 6,8,10,12,14,4$ & $4 / 6$ & $5 / 13$ & 2.300 \\
9 & $1,3,5 / 6,8,10,12,14,4$ & $3 / 6$ & $5 / 6$ & 1.833 \\
10 & $1,3,5 / 2,4$ & $3 / 2$ & $4 / 6$ & $10 / 13$ \\
\hline
\end{tabular}

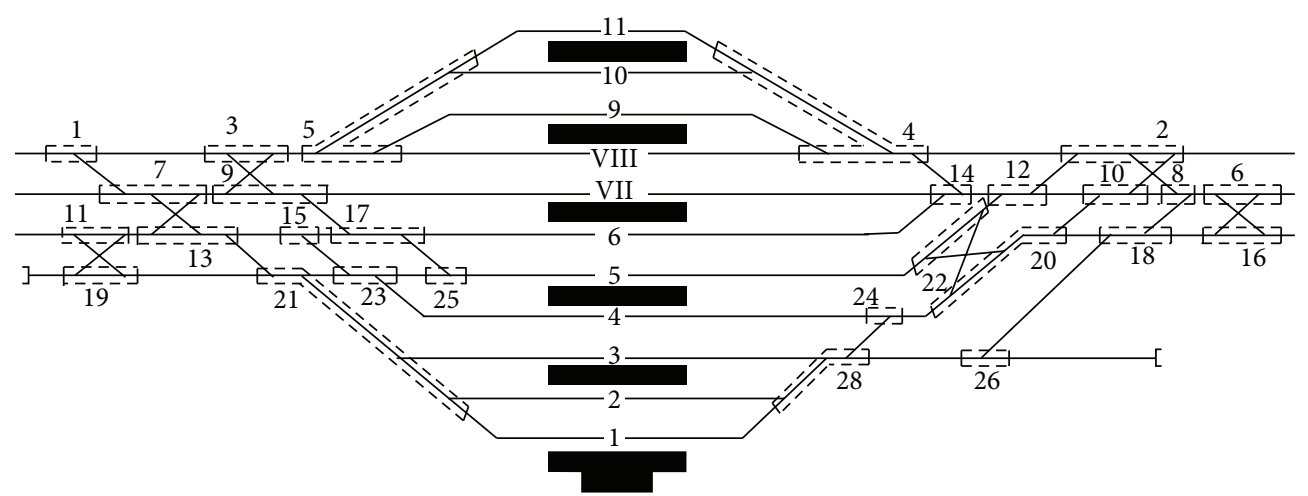

Figure 8: Map of station bottleneck structure (Baoji, China).

2800). $T_{L}$ and $T_{R}$ are calculated each time. It is found that the carrying capacity of the left bottleneck is approximately 1.29 times more than the right bottleneck. Thus, the limited carrying capacity is the right bottleneck section in this railway station.
4.4. Track Unavailable Analysis. From 8:00 to 8:30, the capacity of the station tracks is excessive if there is no track accident. What if the station tracks have a larger area of failures? If the number of unavailable tracks exceeds a certain figure, this will lead to a delay in a train's arrival/departure. 
TABLE 7: Occupation time of each GT (min).

\begin{tabular}{ccccccccccccccc}
\hline GT & 1 & 3 & 5 & 7 & 9 & 11 & 13 & 15 & 17 & 19 & 21 & 23 & 25 & \\
\hline$t_{i j}$ & 1 & 2 & 2 & 3 & 3 & 2 & 3 & 1 & 2 & 2 & 3 & 2 & 1 & \\
\hline GT & 2 & 4 & 6 & 8 & 10 & 12 & 14 & 16 & 18 & 20 & 22 & 24 & 26 & 28 \\
\hline$t_{i j}$ & 3 & 3 & 2 & 2 & 2 & 2 & 2 & 2 & 2 & 3 & 2 & 1 & 1 & 3 \\
\hline
\end{tabular}

TABLE 8: Track allocation results.

\begin{tabular}{lccccccc}
\hline Train & Track & Arrival & Departure & Train & Track & Arrival & Departure \\
\hline 10420 & 9 & $8: 08: 00$ & $8: 19: 00$ & K624 & 9 & $9: 04: 00$ & $9: 12: 00$ \\
10448 & 6 & $8: 08: 00$ & $8: 38: 00$ & K621 & 7 & $9: 04: 00$ & $9: 12: 00$ \\
T23 & 7 & $8: 09: 00$ & $8: 22: 00$ & T75 & 5 & $9: 12: 00$ & $9: 22: 00$ \\
T22 & 5 & $8: 09: 00$ & $8: 22: 00$ & 10452 & 3 & $9: 14: 00$ & $9: 22: 00$ \\
T222 & 1 & $8: 12: 00$ & $8: 22: 00$ & 10454 & 9 & $9: 16: 00$ & $9: 46: 00$ \\
T223 & 10 & $8: 12: 00$ & $8: 22: 00$ & 2669 & 2 & $9: 20: 00$ & $9: 50: 00$ \\
K378 & 10 & $8: 24: 00$ & $8: 31: 00$ & 10175 & 5 & $9: 24: 00$ & $9: 29: 00$ \\
K375 & 9 & $8: 24: 00$ & $8: 31: 00$ & 10176 & 4 & $9: 24: 00$ & $9: 29: 00$ \\
T192 & 1 & $8: 29: 00$ & $8: 33: 00$ & 1486 & 8 & $9: 27: 00$ & $9: 40: 00$ \\
T193 & 7 & $8: 29: 00$ & $8: 33: 00$ & 1147 & 11 & $9: 35: 00$ & $10: 00: 00$ \\
10450 & 2 & $8: 30: 00$ & $9: 00: 00$ & 1150 & 5 & $9: 35: 00$ & $10: 00: 00$ \\
K248 & 10 & $8: 39: 00$ & $8: 49: 00$ & 10456 & 4 & $9: 32: 00$ & $10: 02: 00$ \\
K245 & 1 & $8: 39: 00$ & $8: 49: 00$ & T7 & 6 & $9: 35: 00$ & $9: 45: 00$ \\
D5082 & 6 & $8: 41: 00$ & $9: 11: 00$ & T117 & 7 & $9: 42: 00$ & $9: 52: 00$ \\
D5081 & 10 & $8: 51: 00$ & $9: 21: 00$ & T116 & 3 & $9: 42: 00$ & $9: 52: 00$ \\
\hline
\end{tabular}

Thus, the maximum antijamming capacity in this station is tested. We performed a track unavailable experiment. The results indicate that the maximum number of unavailable tracks at the Baoji station is five from 8:00 to 8:30. Table 11 shows the experimental results.

Table 10 shows that the Baoji station tolerates five unavailable tracks at most. Therefore, we observe the relationships for the number of unavailable tracks, lasting time, and station capacity in Figure 11. There are two parameters related to the size of dispatching trains that have been tested here. It is clear that, with a smaller number of unavailable tracks and shorter lasting time, a larger number of trains tend to be received. As shown in Figure 11, the number of unavailable tracks changes from 6 to 11, the lasting time changes from 5 to $30 \mathrm{~min}$, and the different number of trains that can stay in the station is obtained.

4.5. Convergence Test. To further illustrate the efficiency of the GSA algorithm, a convergence test is given in this case study as shown in Figure 12. The program stops after approximately 30 min with 1000 generations. The result indicates that the algorithm can converge to a steady state. For the real-time usage, operators can set a smaller value of generation or a limited running time. In addition, as the convergence curve indicates, solutions are acceptable when iterations reach onefourth of the total iterations.

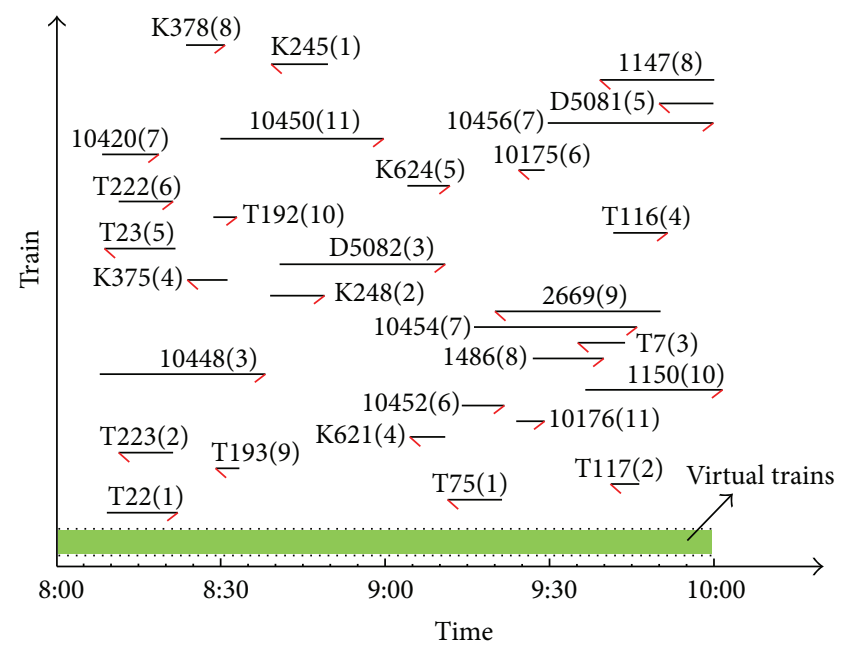

FIgURE 9: Track occupations timeline.

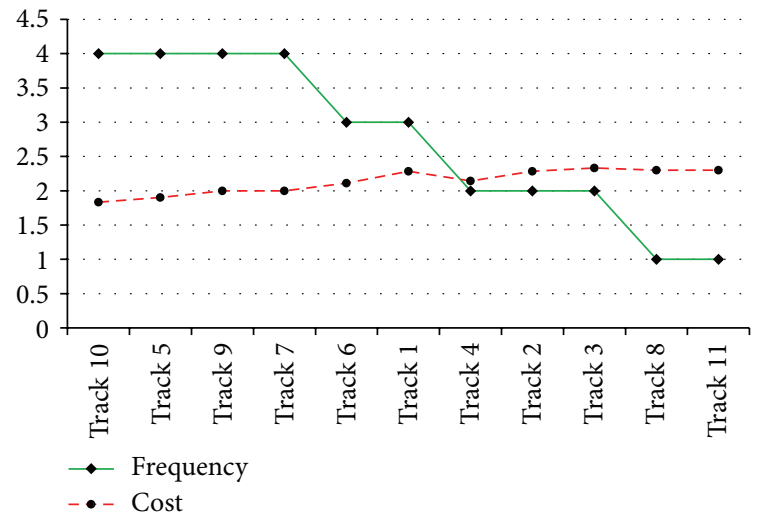

Figure 10: Track occupation frequency and track cost.

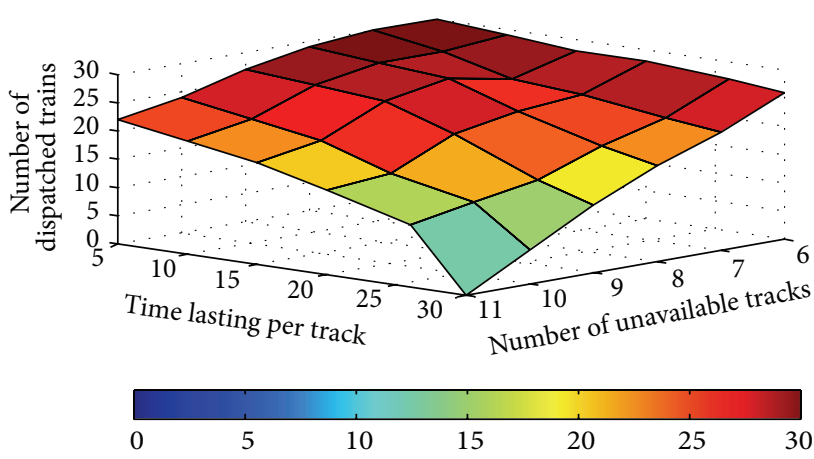

FIGURE 11: Relationships for the number of unavailable tracks, lasting time, and station capacity.

\section{Conclusions}

This paper is motivated by the need to formulate and solve a track reallocation problem in large railway stations. We first explain the key features of using virtual trains to formulate track incidents. A mathematical programming model is developed, which aims at minimizing the total occupation 
TABLE 9: Comparison of optimization results.

\begin{tabular}{|c|c|c|c|c|c|}
\hline \multirow{2}{*}{ Track } & \multirow{2}{*}{ Cost of GTs (min) } & \multicolumn{2}{|c|}{ Optimal results } & \multicolumn{2}{|c|}{ Original results } \\
\hline & & Frequency & Cost (min) & Frequency & Cost (min) \\
\hline 1 & 2.286 & 3 & 6.858 & 3 & 6.858 \\
\hline 2 & 2.286 & 2 & 4.572 & 3 & 6.858 \\
\hline 3 & 2.333 & 2 & 4.666 & 3 & 6.999 \\
\hline 4 & 2.143 & 2 & 4.286 & 3 & 6.429 \\
\hline 5 & 1.9 & 4 & 7.6 & 3 & 5.7 \\
\hline 6 & 2.111 & 3 & 6.333 & 3 & 6.333 \\
\hline 7 & 2 & 4 & 8 & 3 & 6 \\
\hline 8 & 2.3 & 1 & 2.3 & 3 & 6.9 \\
\hline 9 & 2 & 4 & 8 & 2 & 4 \\
\hline 10 & 1.833 & 4 & 7.332 & 2 & 3.666 \\
\hline 11 & 2.3 & 1 & 2.3 & 2 & 4.6 \\
\hline
\end{tabular}

TABLE 10: Bottleneck capacity analysis.

\begin{tabular}{|c|c|c|c|c|c|c|c|c|c|}
\hline \multicolumn{5}{|c|}{ Algorithm parameters } & \multirow{2}{*}{ Track allocations } & \multirow{2}{*}{$T_{L}$} & \multirow{2}{*}{$T_{R}$} & \multirow{2}{*}{$T_{R} / T_{L}$} & \multirow{2}{*}{$T_{L}+T_{R}$} \\
\hline$T_{0}$ & $\tau$ & $\omega$ & $L$ & Run & & & & & \\
\hline 50 & 0.1 & 0.8 & 100 & 2800 & $\begin{array}{c}7,5,8,10,4,1,7,4,2,10,1,10,2,4,9,7 \\
10,2,10,7,1,11,4,10,11,4,3,9,6,10\end{array}$ & 208 & 268 & 1.29 & 476 \\
\hline 30 & 0.1 & 0.8 & 100 & 2600 & $\begin{array}{c}9,6,10,1,5,4,10,2,5,11,7,10,2,1,10 \\
2,4,4,2,11,9,10,4,5,1,2,3,4,10,6\end{array}$ & 219 & 270 & 1.23 & 489 \\
\hline 10 & 0.1 & 0.8 & 100 & 2100 & $\begin{array}{l}10,8,7,1,9,2,10,1,9,11,2,4,10,9,1 \\
4,5,10,4,2,7,10,4,8,11,1,10,4,9,6\end{array}$ & 211 & 280 & 1.33 & 491 \\
\hline 50 & 0.5 & 0.8 & 100 & 2100 & $\begin{array}{c}10,7,5,2,8,4,4,10,8,2,6,11,7,1,4,9 \\
10,6,11,1,7,10,4,5,2,4,8,6,10,9\end{array}$ & 223 & 281 & 1.26 & 504 \\
\hline 50 & 1 & 0.8 & 100 & 1800 & $\begin{array}{c}6,4,10,11,1,5,10,5,9,11,1,10,9,2,7 \\
5,10,6,8,5,3,10,7,11,2,1,4,7,10,6\end{array}$ & 223 & 283 & 1.27 & 506 \\
\hline 50 & 0.1 & 0.5 & 100 & 900 & $\begin{array}{l}10,11,4,5,7,1,8,5,7,10,6,10,7,8,7 \\
5,4,10,8,1,4,10,6,2,6,11,10,9,2,8\end{array}$ & 226 & 283 & 1.25 & 509 \\
\hline 50 & 0.1 & 0.2 & 100 & 400 & $\begin{array}{c}9,7,10,5,11,1,3,1,9,10,5,11,7,3,1,8 \\
10,4,10,7,8,5,6,10,2,5,1,6,10,3\end{array}$ & 223 & 290 & 1.30 & 513 \\
\hline 50 & 0.1 & 0.8 & 50 & 1400 & $\begin{array}{c}5,4,10,3,7,2,10,11,5,1,6,10,3,7,10 \\
9,11,4,5,11,8,10,5,2,7,10,3,1,4,2\end{array}$ & 223 & 284 & 1.27 & 507 \\
\hline 50 & 0.1 & 0.8 & 30 & 840 & $\begin{array}{c}9,6,7,5,1,10,10,9,1,7,2,10,1,6,10 \\
9,7,5,3,9,2,5,4,8,11,5,4,6,7,3 \\
\end{array}$ & 217 & 294 & 1.35 & 511 \\
\hline 10 & 1 & 0.2 & 30 & 60 & $\begin{array}{c}7,1,8,9,11,5,3,7,5,2,9,4,6,1,7,5,2 \\
9,1,6,4,10,8,9,5,10,1,11,8,3\end{array}$ & 230 & 306 & 1.33 & 536 \\
\hline \multicolumn{4}{|c|}{ Average } & 1500 & - & 220 & 284 & 1.29 & 504 \\
\hline
\end{tabular}

TABLE 11: Test of the number of unavailable tracks.

\begin{tabular}{lcc}
\hline Track unavailable & Track allocation & $\begin{array}{c}\text { Occupation } \\
\text { time of GTs }\end{array}$ \\
\hline 1 & $10,5,9,7,6,4,10,5,9,7,6$ & 21.831 \\
1,2 & $10,5,9,7,6,4,10,5,9,7,6$ & 21.831 \\
$1,2,3$ & $10,5,9,7,6,4,10,5,9,7,6$ & 21.831 \\
$1,2,3,4$ & $10,5,9,7,6,8,10,5,9,7,6$ & 21.988 \\
$1,2,3,4,5$ & $10,9,7,6,8,11,10,9,7,6,8$ & 22.788 \\
$1,2,3,4,5,6$ & Reallocation failure & Train delay \\
\hline
\end{tabular}

time of station bottleneck sections to avoid train delays. In addition, a hybrid algorithm between the genetic algorithm and the simulated annealing algorithm is designed. The case study for the Baoji railway station in China verifies the efficiency of the proposed model and the algorithm.

Our future study will focus on (1) developing different optimization or reformulation methods that can reduce the CPU time, improving the solution efficiency, and (2) modeling different scenarios for station incidents, for example, signal problems and switching problems, to address problems in railway station operations.

\section{Conflict of Interests}

The authors declare that there is no conflict of interests regarding the publication of this paper. 


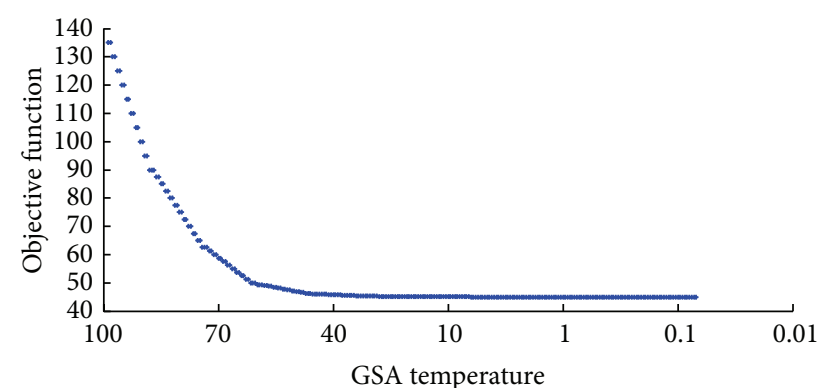

FIGURE 12: Convergence test.

\section{Acknowledgments}

This paper is supported by the NSFC (71473259, 71390332), the Fundamental Research Funds for the Central Universities (2015YJS101), and the Specialized Research Fund for the Doctoral Program of Higher Education (20130009110001).

\section{References}

[1] A. Caprara, M. Fischetti, and P. Toth, "Modeling and solving the train timetabling problem," Operations Research, vol. 50, no. 5, pp. 851-861, 2002.

[2] P. J. Zwaneveld, L. G. Kroon, and S. P. M. Van Hoesel, "Routing trains through a railway station based on a node packing model," European Journal of Operational Research, vol. 128, no. 1, pp. 14-33, 2001.

[3] P. J. Zwaneveld, L. G. Kroon, H. E. Romeijn et al., "Routing trains through railway stations: model formulation and algorithms," Transportation Science, vol. 30, no. 3, pp. 181-194, 1996.

[4] D. D. L. Cardillo and N. Mione, " $k L$-list $\lambda$ colouring of graphs," European Journal of Operational Research, vol. 106, no. 1, pp. 160-164, 1998.

[5] A. Billionnet, "Using integer programming to solve the trainplatforming problem," Transportation Science, vol. 37, no. 2, pp. 213-222, 2003.

[6] M. Carey and S. Carville, "Scheduling and platforming trains at busy complex stations," Transportation Research Part A: Policy and Practice, vol. 37, no. 3, pp. 195-224, 2003.

[7] L. J. Kang, J. J. Wu, and H. J. Sun, "Using simulated annealing in a bottleneck optimization model at railway stations," Journal of Transportation Engineering, vol. 138, no. 11, pp. 1396-1402, 2012.

[8] F. Barber, M. A. Salido, L. P. Ingolotti, M. Abril, A. L. Lova, and M. P. Tormos, "An interactive train scheduling tool for solving and plotting running maps," in Current Topics in Artificial Intelligence, vol. 3040 of Lecture Notes in Computer Science, pp. 646-655, Springer, Berlin, Germany, 2004.

[9] A. D’Ariano, D. Pacciarelli, and M. Pranzo, "Assessment of flexible timetables in real-time traffic management of a railway bottleneck," Transportation Research Part C: Emerging Technologies, vol. 16, no. 2, pp. 232-245, 2008.

[10] M. Heydar, M. E. H. Petering, and D. R. Bergmann, "Mixed integer programming for minimizing the period of a cyclic railway timetable for a single track with two train types," Computers \& Industrial Engineering, vol. 66, no. 1, pp. 171-185, 2013.
[11] S. Narayanaswami and N. Rangaraj, "Modelling disruptions and resolving conflicts optimally in a railway schedule," Computers \& Industrial Engineering, vol. 64, no. 1, pp. 469-481, 2013.

[12] Y.-H. Cheng and Y.-C. Tsai, "Railway-controller-perceived competence in incidents and accidents," Ergonomics, vol. 54, no. 12, pp. 1130-1146, 2011.

[13] F. Belmonte, W. Schön, L. Heurley, and R. Capel, "Interdisciplinary safety analysis of complex socio-technological systems based on the functional resonance accident model: an application to railway traffic supervision," Reliability Engineering and System Safety, vol. 96, no. 2, pp. 237-249, 2011.

[14] B. L. Hollis and P. J. Green, "Real-life vehicle routing with time windows for visual attractiveness and operational robustness," Asia-Pacific Journal of Operational Research, vol. 29, no. 4, Article ID 1250017, 2012.

[15] X. Gandibleux, X. Delorme, and V. T'Kindt, "An ant colony optimisation algorithm for the set packing problem," in Ant Colony Optimization and Swarm Intelligence, vol. 3172 of Lecture Notes in Computer Science, pp. 49-60, Springer, Berlin, Germany, 2004.

[16] X. Gandibleux, J. Jorge, S. Angibaud, X. Delorme, and J. Rodriguze, "An ant colony optimization inspired algorithm for the set packing problem with application to railway infrastructure," in Proceedings of the 6th Metaheuristics International Conference, pp. 309-396, Vienna, Austria, 2005.

[17] G. Caimi, D. Burkolter, and T. Herrmann, "Finding delaytolerant train routings through stations," in Operations Research Proceedings 2004, vol. 2004 of Operations Research Proceedings, pp. 136-143, Springer, Berlin, Germany, 2005.

[18] T. M. Herrmann, Stability of timetables and train routing the station regions [Ph.D. thesis], Swiss Federal Institute of Technology, Zürich, Switzerland, 2006.

[19] J. J. Wu, L. J. Kang, H. J. Sun, and X. L. Jia, “Track allocation optimization in railway station: mean-variance model and case study," Journal of Transportation Engineering, vol. 139, no. 5, pp. 540-547, 2013.

[20] F. Zhao and X. G. Zeng, "Simulated annealing-genetic algorithm for transit network optimization," Journal of Computing in Civil Engineering, vol. 20, no. 1, pp. 57-68, 2006.

[21] L. J. Kang, X. N. Zhu, J. J. Wu, H. J. Sun, S. Siriya, and K. Kanokvate, "Departure time optimization of last trains in subway networks: mean-variance model and GSA algorithm," Journal of Computing in Civil Engineering, Article ID 04014081, pp. 1-12, 2014.

[22] C. Koulamas, S. R. Antony, and R. Jaen, "A survey of simulated annealing applications to operations research problems," Omega, vol. 22, no. 1, pp. 41-56, 1994.

[23] C. R. Reeves, "Genetic algorithms for the operations researcher," INFORMS Journal on Computing, vol. 9, no. 3, pp. 231-250, 1997.

[24] P. Sels, P. Vansteenwegen, T. Dewilde, D. Cattrysse, B. Waquet, and A. Joubert, "The train platforming problem: the infrastructure management company perspective," Transportation Research Part B: Methodological, vol. 61, pp. 55-72, 2014. 


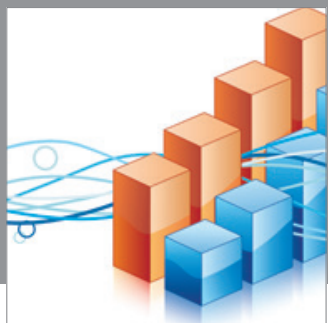

Advances in

Operations Research

mansans

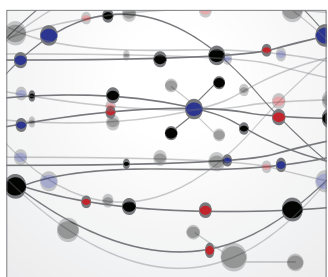

The Scientific World Journal
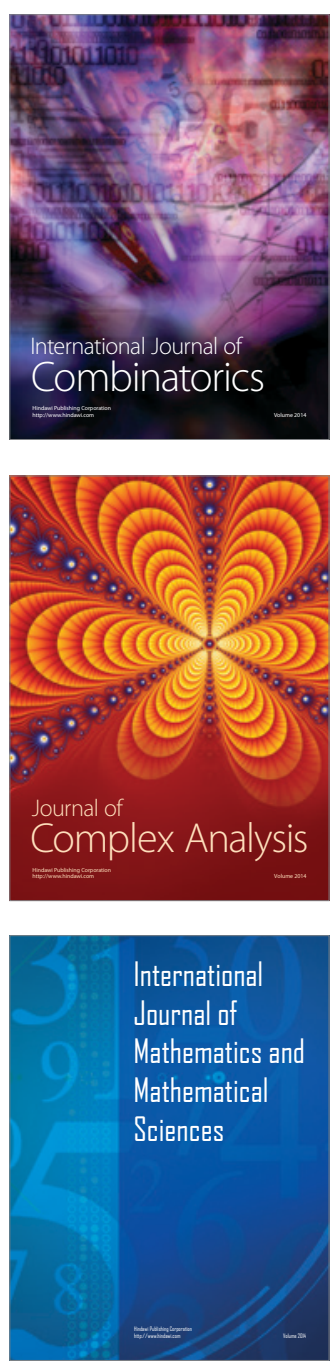
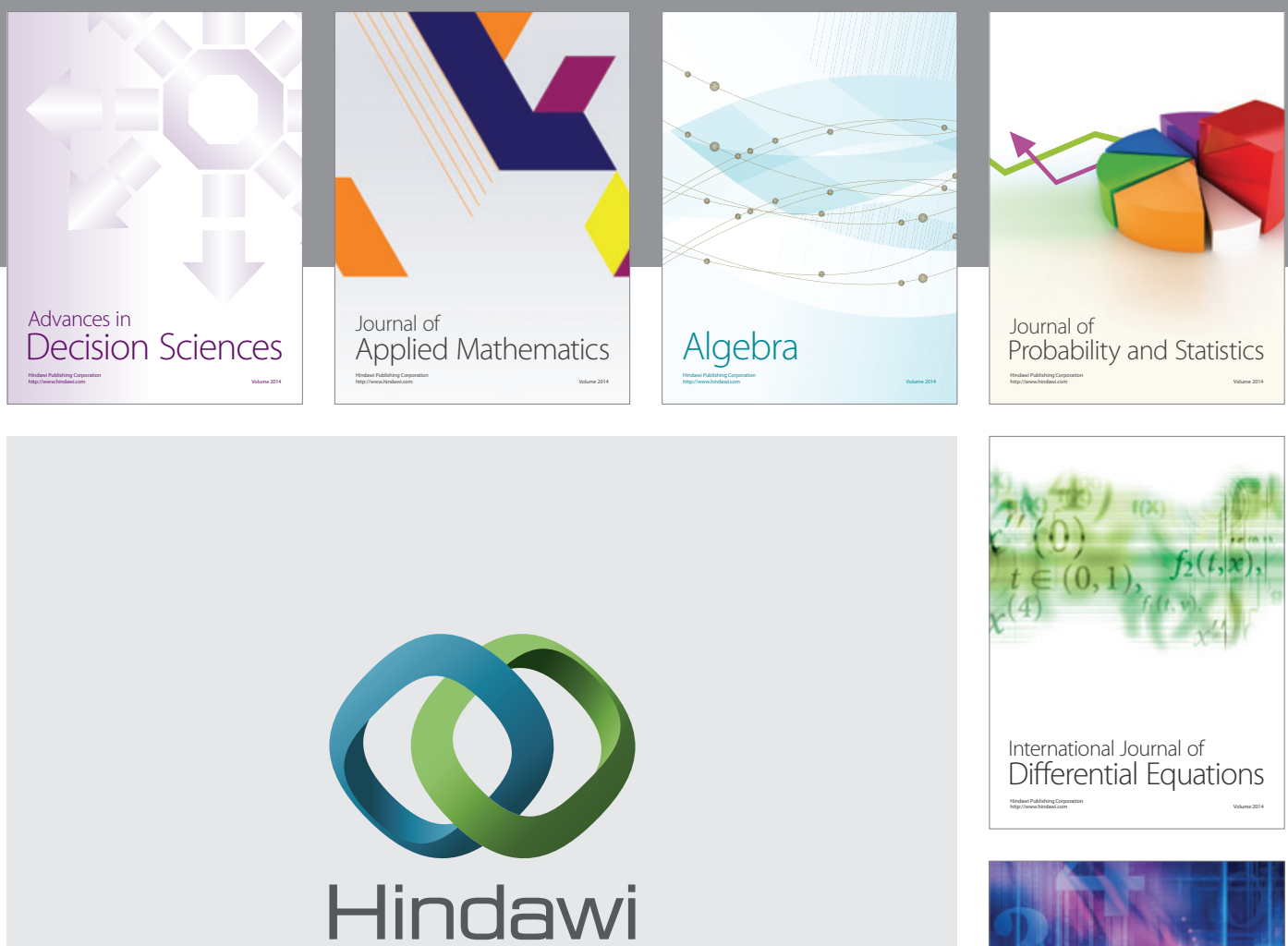

Submit your manuscripts at http://www.hindawi.com
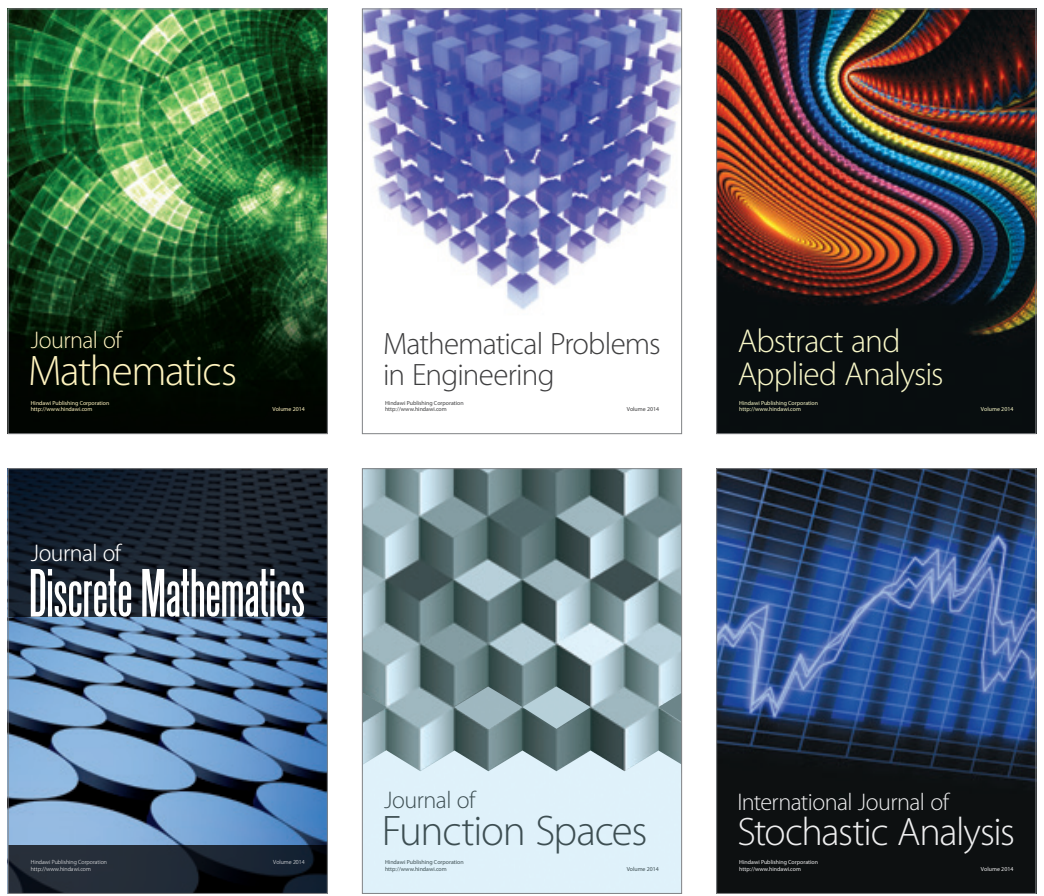

Journal of

Function Spaces

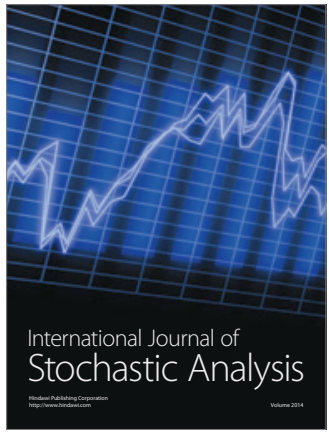

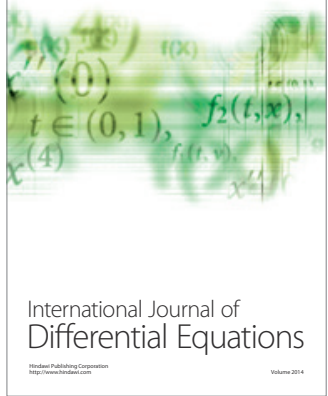
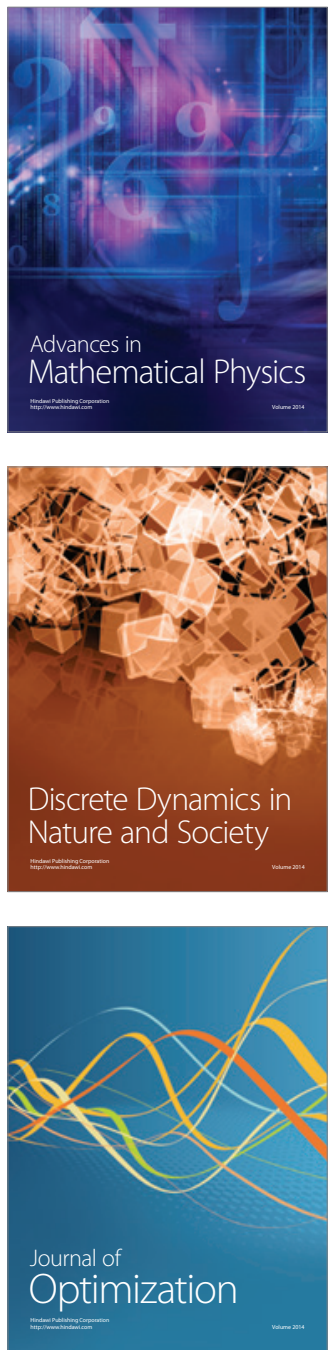